\title{
Setting up a new old age psychiatry service
}

\author{
General practitioner views on the priorities
}

\author{
Michael Kirby and Colm Cooney
}

\begin{abstract}
The views of general practitioners (GP3) on the priorities for a new old age psychiatry service were obtained. Most GPs identified dementia patients with behavioural or psychiatric disturbance as being the greatest priority for an old age psychiatry service. There was less consensus on the priorities for dellvery of the service, but hosplital-based services such as in-patient and day hospltal facilities remain high on the GPs' priority list. There was a widely-based desire for results of ascessments to be communicated by telephone, followed later by lefter. These findings have been taken into account in the development of our new service.
\end{abstract}

The development of old age psychiatry services in the Republic of Ireland are at a relatively early stage. Prior to 1996 there were only two dedicated old age psychiatry services in the city of Dublin, serving two specific catchment areas, with the elderly mentally ill in the rest of Dublin being catered for by general adult psychiatry. A new old age psychiatry service for south-east Dublin was established in 1996.

The views of general practitioners (GPs) deserve close attention for a variety of reasons. Over $95 \%$ of mental illness is managed in the community (Goldberg \& Huxley, 1992). The elderly have a high GP consultation rate and the GP is in an ideal position to, at least, advise on the psychiatric needs of older people. Most old age psychiatry services are community oriented and demand close GP involvement. Furthermore, the institution of the purchaser/provider system in the UK (while not currently an issue for the Irish health service) emphasises the importance of old age psychiatry services being sensitive to the views of GPs (Banerjee et al, 1993). On the other hand, old age psychiatry services obviously cannot take over all the elderly with mental health problems in the community and must work in conjunction with GPs to prioritise the available resources. This is of particular relevance in the development of our new old age psychiatry service in south-east Dublin with its large catchment area population of 29000 people over 65 years and a particularly large proportion of over 75s.

Banerjee et al (1993) investigated service contacts between psychogeriatricians and GPs, but from the perspective of the psychogeriatrician rather than the GP. Ashaye et al (1996) and Eastley \& Nowers (1997) examined the satisfaction of GPs with existing psychogeriatric services but did not ascertain GP views on specific service priorities. The development of a new old age psychiatry service in an area which did not previously have access to a specialist service for the elderly mentally ill afforded us the opportunity to ascertain GP views on the needs and priorities for such a service, as opposed to examining the deficiencies in, or satisfaction with, an existing old age psychiatry service.

\section{The study}

All GPs in a psychiatric catchment area were identified from a list provided by community care services. This catchment area did not, at the time of the survey, have a specialist old age psychiatry service and the elderly were catered for within the general adult mental health services.

The questionnaire was designed with the intention of identifying GP views on the needs for psychiatric services for their elderly mentally ill patients. The GP was asked to prioritise patient groups (dementia with behavioural disturbance, paranoid disorders, depression, anxiety disorders and alcohol problems) and also to prioritise the type of services (domiciliary, day hospital, in-patient, out-patient clinic, respite and extended care services) required. The questions were framed in this manner to avold the risk of 'high priority' being assigned to all or most items. The questionnaire also contained items on preferred method of communication of results of psychiatric assessments on the elderly, on the desirability of having a meeting to discuss cases, and on basic practice details. 
The questionnaire was piloted on five GPs working in the Dublin area and alterations made following their comments. The final version was sent to all 99 GPs working in the catchment area. Those who had not replied after eight weeks were sent a second copy.

\section{Findings}

Sixty replies were received, giving an overall response rate of $61 \%$. Twenty-three $(38 \%)$ GPs indicated that they worked in single-handed practices. The mean number of doctors per practice was 2.4 (range 1 to 7). The mean patient list size (including private patients) was 1655 . The percentage of their patient population over 65 years was estimated to be less than $10 \%$ by 11 (18\%) GPs, between 10 and $20 \%$ by 28 GPs $(47 \%)$ and more than $20 \%$ by 19 GPs (32\%) (two GPs did not state a percentage). Forty-five GPs (75\%) said that they referred five or less patients of 65 years or older per year to the existing general adult psychiatric service.

Thirty-eight GPs (63\%) believed that dementia patients with behavioural/psychiatric complications were the patient group in greatest need of old age psychiatry services. Six GPs (10\%) identified those with paranoid disorders as the highest priority, five GPs $(8 \%)$ depressive disorders, two GPs $(3 \%)$ patients with anxiety disorders and a further two GPs (3\%) alcohol problems (seven GPs did not prioritise, in most instances marking all patients' groups as the 'highest priority'). Given the large majority who identify psychiatric services for dementia as the greatest need, looking only at the highest priority may underestimate the relative priorities GPs attach to the other conditions. Consequently, we attributed 'high priority' to those patient groups identified by the GPs as either the highest or second highest priority for old age psychiatry services. Analysing the replies in this manner, 'high priority' was assigned to dementia patients by 43 GPs $(72 \%)$, those with paranoid disorders by 27 GPs (45\%), depressed elderly by 22 GPs (37\%), and those with anxiety disorders or with alcohol problems by seven GPs (12\%) each.

There were widely spread views on the issue of priorities for the manner of delivery of an old age psychiatry service. Twelve GPs $(20 \%)$ felt that the availability of day hospital services was the greatest priority, $11(18 \%)$ identified domiciliary assessment, $10(16 \%)$ in-patient services, $10(17 \%)$ out-patient clinic services, nine $(15 \%)$ extended care and three $(5 \%)$ respite care (six GPs did not prioritise). Again, we looked at 'high priority' services, those assigned highest or second highest priority by the GPs. High priority was given to day hospital services by 26 GPs (43\%), in-patient services by 26 (43\%), domiciliary assessment by 19 (32\%), outpatient clinic services by $16(27 \%)$, extended care by $13(22 \%)$ and respite care nine (15\%).

Current practice for feedback to the GP following psychiatric assessment of their older patients by the general adult psychiatric service was usually letter alone according to 56 GPs (93\%). However, 42 GPs $(70 \%)$ expressed a preference for telephone call followed by letter as the manner of communication of assessment results. Sixteen $(27 \%)$ only wished to be contacted by letter, while none opted for telephone communication alone (two GPs expressed no preference). On the specific issue of a meeting with the old age psychiatry team to discuss a patient 'in certain cases', 43 (72\%) replied positively.

\section{Comment}

The reported number of referrals of older people to the general adult psychiatric services was low. with only $25 \%$ of GPs referring more than five patients per year compared to $52 \%$ in Ashaye et als (1996) study of an existing psychogeriatric service in Barnet, UK. While many other issues, including practice population, influence the number of referrals, the lack of a specialist old age psychiatric service in the catchment area studied may be a factor in the low GP referrals. Indeed, this comment was made in a number of replies.

Dementia patients with behavioural or psychiatric disturbance were clearly identified by the GPs as those in greatest need of old age psychiatry services. This would appear to reflect GP awareness of the immense impact of these symptoms on the quality of life of the elderly person with dementia and of the carer, and the difficulty the GP may experience in managing these problems. It is not unusual for conflict to occur between geriatric medicine and psychiatry with regard to responsibilities for patients with dementia. Furthermore, psychiatric services which are not specifically dedicated to the care of the elderly mentally ill may be more reluctant to take on dementia patients than the elderly with functional disorders, such as depression. It is essential that specialist old age psychiatry services are responsive to the needs of dementia patients with behavioural/psychiatric symptoms, and can offer primary care an area of expertise which may not be readily available within general adult services. Anxiety disorders and alcohol problems among the elderly received lowest priority ratings for psychiatry service need and, by implication, GPs would appear to believe that they should be catered for by primary care and allied services. 
There was a wide variety of views among the GPs as to what the priorities should be in terms of delivery of old age psychiatry services. Nevertheless, high priority was given predominantly to more acute services compared to respite and extended care services. There can be a perception within old age psychiatry services that the main concern of primary care, and our medical and surgical hospital-based colleagues, is the 'placement' of dementia patients. This would not appear to be substantiated in this study. Old age psychiatry services generally place a large emphasis on domiciliary assessment and management and 'high priority' can be attributed to domiciliary assessment from the views of $32 \%$ of GPs. However, in-patient and day hospital assessment generate 'high priority' rating in the replies of a larger proportion of GPs, $43 \%$ in each case. The availability of in-patient and day hospital services for their elderly patients with mental health problems rates highly in GPs priorities.

Good communication between the old age psychiatrist and the GP is essential for adequate assessment and management of older people with mental health problems and also in view of the considerable comorbidity of medical and psychiatric conditions in the elderly. The clear preference of the GPs in this study was for both telephone and written communication of the results of assessments. The desire of GPs for telephone contact from the psychiatrist, and their dissatisfaction at the lack of such, has previously been emphasised in the context of discharge from in-patient care (Wylie, 1994). GP views on the importance of close communication with the psychiatric team and continuing involvement in the care of their elderly patients with mental disorders was further underlined by the wish, expressed by $72 \%$ of GPs, to have a meeting with the team in 'certain cases'.

We have endeavoured to utilise the findings of this study in the development of our old age psychiatry service. Our intention has been to establish a hospital-based but communityoriented service with the close involvement of primary care (for example, we ask our GP colleagues to prescribe for the patients in the community following assessment by the service). Therefore, it is reassuring that the GPs expressed a desire for close communication with the service. We have instituted a policy of contacting the patient's GP by telephone after the first assessment and subsequently if there is to be a significant change in the management plan. In general telephone contact is made by our secretarial staff and, as a relatively brief discussion between the psychiatrist and the GP is usually sufficient, this practice does not have major workload implications for the psychiatrist. We have found that telephone communication facilitates quick implementation of management plans and helps to maintain close GP involvement with the patient after referral.

\section{Acknowledgement}

We thank our general practitioner colleagues for their cooperation in this study.

\section{References}

Ashaye, O., Dhadphale, M. \& OKore, A. (1996) General practitioners' views of a psychogeriatric service. Psychiatric Bulletin, 20. 140-142.

BANERJEe. S., LINDESAY, J. \& MURPHY, E. (1993) Psychogeriatricians and general practitioners: a national survey. Psychiatric Bulletin, 17, 592-594.

EASTLEY. R. \& NOWERS. M. (1997) A community mental health team for the elderly: a survey of GPs' views on the service. Psychiatric Bulletin. 21, 88-90.

GOLDBERG, D. \& HuXLEY, P. (1992) Common Mental Disorders: A Bio-Social Model. London: Routledge.

WruE. A. S. (1994) General practitioners' involvement in psychiatric patients' discharge plans. Psychiatric Bulletin, 18, 207-208.

*Michael Kirby, Senior Registrar in Old Age Psychiatry and Colm Cooney, Consultant in Old Age Psychiatry, St Vincent's Hospital, Elm Park, Dublin 4, Ireland

*Correspondence 